\title{
Cumulative Results of Irradiation
} Induced Creep of Material IG-110 - Prepared for Toyo Tanso Co., Ltd.

Approved for public release.

Distribution is unlimited.
Anne A. Campbell

Yutai Katoh

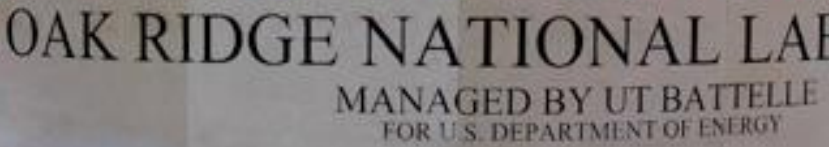




\section{DOCUMENT AVAILABILITY}

Reports produced after January 1, 1996, are generally available free via US Department of Energy (DOE) SciTech Connect.

Website http://www.osti.gov/scitech/

Reports produced before January 1, 1996, may be purchased by members of the public from the following source:

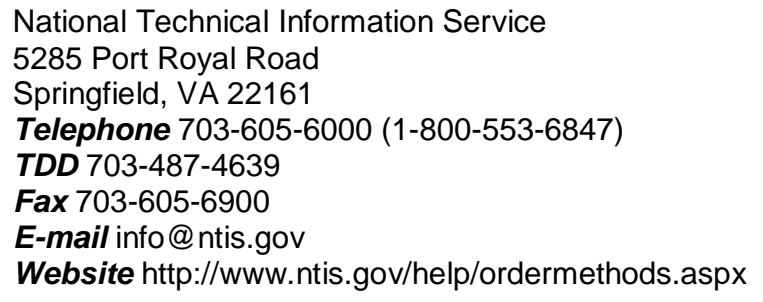

Reports are available to DOE employees, DOE contractors, Energy Technology Data Exchange representatives, and International Nuclear Information System representatives from the following source:

Office of Scientific and Technical Information

PO Box 62

Oak Ridge, TN 37831

Telephone 865-576-8401

Fax 865-576-5728

E-mail reports@osti.gov

Website http://www.osti.gov/contact.html

This report was prepared by UT-BATTELLE, LLC, (UT-Battelle) on behalf of the U.S. Department of Energy (DOE), as an account of work sponsored by TOYO TANSO CO., Ltd. Neither UT-Battelle, DOE, the U. S. Government, or any person acting on their behalf: (a) makes any warranty or representation, express or implied, with respect to the information contained in this report; or (b) assumes any liabilities with respect to the use of, or damages resulting from the use of any information contained in the report. 
Irradiation effects study of Toyo Tanso HTGR Graphite, Part II: Irradiation and Post-irradiation examination

DOE Project No. NFE-10-02974

\title{
CUMULATIVE RESULTS OF IRRADIATION INDUCED CREEP OF MATERIAL IG-110 - PREPARED FOR TOYO TANSO CO., LTD.
}

Anne A. Campbell

Yutai Katoh

Date Published: July 2018

\author{
Prepared by \\ OAK RIDGE NATIONAL LABORATORY \\ Oak Ridge, Tennessee 37831-6283 \\ managed by \\ UT-BATTELLE, LLC \\ for the \\ US DEPARTMENT OF ENERGY \\ under contract DE-AC05-00OR22725
}





\section{ACKNOWLEDGEMENT}

This program would not have been completed without the expertise and input from numerous persons throughout the laboratory. Special notice needs to be given to the staff of the LAMDA laboratory: Joshua Schmidlin, Patricia Tedder, Stephanie Curlin, Michael McAllister, Bill Comings, Brian Eckhart, J. Felipe Mora, and Wallace Porter.

Research sponsored by Toyo Tanso Co., Ltd. under contract NFE-10-02974 with UT-Battelle, LLC. A portion of this research at ORNL's High Flux Isotope Reactor was sponsored by the Scientific User Facilities Division, Office of Basic Energy Sciences, U.S. Department of Energy. Oak Ridge National Laboratory is managed by UT-Battelle, LLC under Contract No. DE-AC0500OR22725 for the U.S. Department of Energy. 



\section{INTRODUCTION}

This letter report provides additional results from the irradiation creep section of the ORNL report number ORNL/TM-2017/705 [1]. The results presented include the medium and high fluence dimensional change of stressed and unstressed specimens.

\section{RESULTS}

The post-irradiation examination of the first, of three irradiation-induced creep experiments, was completed, and the preliminary results are summarized in this section. The creep experiment had two distinct temperature regions: $300^{\circ} \mathrm{C}$, and $600^{\circ} \mathrm{C}$. The applied compressive stress was nominally $13.5 \mathrm{MPa}$. Unlike the rabbits, the design of these experiments was very complex. The final average specimen temperatures achieved for these regions were $243^{\circ} \mathrm{C}$ and $497^{\circ} \mathrm{C}$, respectively. The results of the dimensional change of the stressed specimens are shown in Figure 1. The individual specimen results are listed in Table 1.

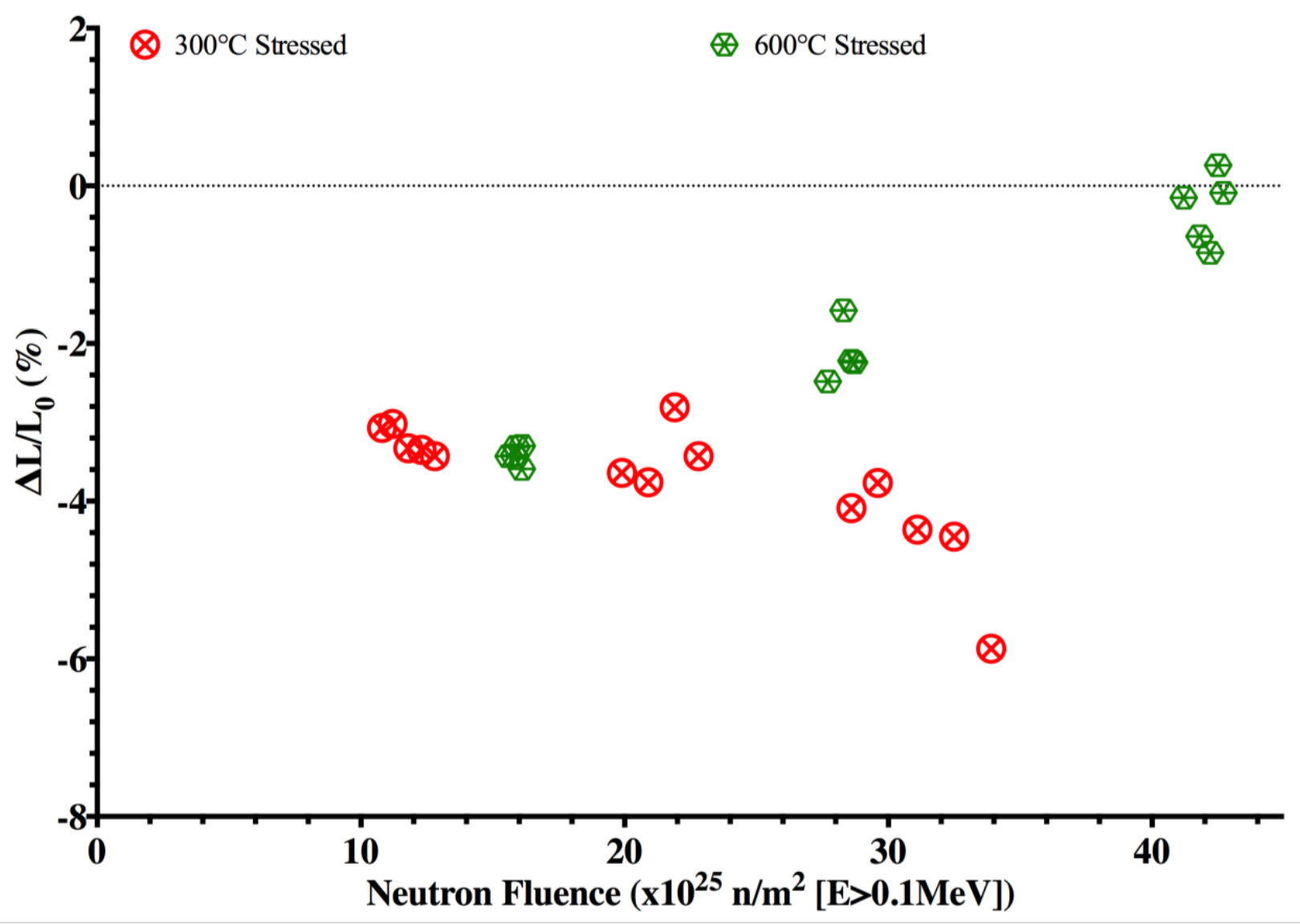

Figure 1. Dimensional strain of stressed specimens in first creep experiment. 
Table 1. Individual specimen results.

\begin{tabular}{|c|c|c|c|c|c|c|c|c|}
\hline ID & Material & $\begin{array}{c}\text { Sample } \\
\text { Type }\end{array}$ & Orien. & Creep & $\begin{array}{c}\text { Design } \\
\text { Temp. }\left({ }^{\circ} \mathrm{C}\right)\end{array}$ & $\begin{array}{c}\text { Measured } \\
\text { Temp. }\left({ }^{\circ} \mathbf{C}\right)\end{array}$ & $\begin{array}{c}\text { Neutron } \\
\text { Fluence } \\
\left(\mathbf{x 1 0} \mathbf{n}^{25} / \mathbf{m}^{2}\right. \\
{[\mathrm{E}>0.1} \\
\mathrm{MeV}]) \\
\end{array}$ & $\begin{array}{l}\text { Length } \\
\text { Change } \\
\left(\Delta \mathrm{L} / \mathrm{L}_{0}\right)\end{array}$ \\
\hline T14A38 & IG110 & CRP & $\mathrm{AX}$ & Stressed & 300 & 243 & 10.8 & $-3.07 \%$ \\
\hline T14A37 & IG110 & CRP & $\mathrm{AX}$ & Stressed & 300 & 243 & 11.2 & $-3.02 \%$ \\
\hline T13A33 & IG110 & CRS & $\mathrm{AX}$ & Stressed & 300 & 243 & 11.8 & $-3.33 \%$ \\
\hline T14A36 & IG110 & CRP & $\mathrm{AX}$ & Stressed & 300 & 244 & 12.3 & $-3.35 \%$ \\
\hline T14A35 & IG110 & CRP & $\mathrm{AX}$ & Stressed & 300 & 253 & 12.8 & $-3.43 \%$ \\
\hline T14A49 & IG110 & CRP & $\mathrm{AX}$ & Stressed & 300 & 226 & 19.9 & $-3.64 \%$ \\
\hline T13A21 & IG110 & CRS & $\mathrm{AX}$ & Stressed & 300 & 226 & 20.9 & $-3.76 \%$ \\
\hline T14A48 & IG110 & CRP & $\mathrm{AX}$ & Stressed & 300 & 227 & 21.9 & $-2.81 \%$ \\
\hline T14A47 & IG110 & CRP & $\mathrm{AX}$ & Stressed & 300 & 235 & 22.8 & $-3.43 \%$ \\
\hline T14A74 & IG110 & CRP & $\mathrm{AX}$ & Stressed & 300 & 240 & 28.6 & $-4.09 \%$ \\
\hline T14A73 & IG110 & CRP & $\mathrm{AX}$ & Stressed & 300 & 241 & 29.6 & $-3.77 \%$ \\
\hline $\mathrm{T} 13 \mathrm{~A} 27$ & IG110 & CRS & $\mathrm{AX}$ & Stressed & 300 & 241 & 31.1 & $-4.36 \%$ \\
\hline T14A62 & IG110 & CRP & $\mathrm{AX}$ & Stressed & 300 & 242 & 32.5 & $-4.45 \%$ \\
\hline T14A34 & IG110 & CRP & $\mathrm{AX}$ & Stressed & 300 & 251 & 33.9 & $-5.87 \%$ \\
\hline T14A40 & IG110 & CRP & $\mathrm{AX}$ & Unstressed & 300 & 238 & 12.2 & $1.28 \%$ \\
\hline T14A39 & IG110 & CRP & $\mathrm{AX}$ & Unstressed & 300 & 239 & 12.6 & $1.69 \%$ \\
\hline T13A34 & IG110 & CRS & $A X$ & Unstressed & 300 & 245 & 13.0 & $1.05 \%$ \\
\hline T14A55 & IG110 & CRP & $\mathrm{AX}$ & Unstressed & 300 & 240 & 21.7 & $1.39 \%$ \\
\hline T14A54 & IG110 & CRP & $\mathrm{AX}$ & Unstressed & 300 & 241 & 22.4 & $1.43 \%$ \\
\hline T13A67 & IG110 & CRS & $\mathrm{AX}$ & Unstressed & 300 & 248 & 23.1 & $0.47 \%$ \\
\hline T14A63 & IG110 & CRP & $\mathrm{AX}$ & Unstressed & 300 & 256 & 32.3 & $2.52 \%$ \\
\hline T14A64 & IG110 & CRP & $\mathrm{AX}$ & Unstressed & 300 & 257 & 33.4 & $2.57 \%$ \\
\hline T13A28 & IG110 & CRS & $\mathrm{AX}$ & Unstressed & 300 & 264 & 34.4 & $1.93 \%$ \\
\hline $\mathrm{T} 14 \mathrm{~A} 72$ & IG110 & CRP & $\mathrm{AX}$ & Stressed & 600 & 496 & 15.6 & $-3.43 \%$ \\
\hline T14A71 & IG110 & CRP & $A X$ & Stressed & 600 & 500 & 15.8 & $-3.42 \%$ \\
\hline T13A31 & IG110 & CRS & $\mathrm{AX}$ & Stressed & 600 & 501 & 15.9 & $-3.31 \%$ \\
\hline $\mathrm{T} 14 \mathrm{~A} 70$ & IG110 & CRP & $\mathrm{AX}$ & Stressed & 600 & 502 & 16.1 & $-3.59 \%$ \\
\hline T14A69 & IG110 & CRP & $\mathrm{AX}$ & Stressed & 600 & 505 & 16.1 & $-3.30 \%$ \\
\hline T14A45 & IG110 & CRP & $A X$ & Stressed & 600 & 515 & 27.7 & $-2.48 \%$ \\
\hline T13A19 & IG110 & CRS & $A X$ & Stressed & 600 & 521 & 28.3 & $-1.58 \%$ \\
\hline T14A01 & IG110 & CRP & $\mathrm{AX}$ & Stressed & 600 & 522 & 28.6 & $-2.22 \%$ \\
\hline $\mathrm{T} 14 \mathrm{~A} 00$ & IG110 & CRP & $\mathrm{AX}$ & Stressed & 600 & 524 & 28.7 & $-2.24 \%$ \\
\hline T14A60 & IG110 & CRP & $\mathrm{AX}$ & Stressed & 600 & 481 & 41.2 & $-0.15 \%$ \\
\hline T14A53 & IG110 & CRP & $\mathrm{AX}$ & Stressed & 600 & 485 & 41.8 & $-0.64 \%$ \\
\hline T13A25 & IG110 & CRS & $A X$ & Stressed & 600 & 486 & 42.2 & $-0.85 \%$ \\
\hline T14A52 & IG110 & CRP & $\mathrm{AX}$ & Stressed & 600 & 487 & 42.5 & $0.26 \%$ \\
\hline T14A51 & IG110 & CRP & $\mathrm{AX}$ & Stressed & 600 & 489 & 42.7 & $-0.09 \%$ \\
\hline T14A44 & IG110 & CRP & $\mathrm{AX}$ & Unstressed & 600 & 488 & 16.0 & $-1.84 \%$ \\
\hline T14A43 & IG110 & CRP & $A X$ & Unstressed & 600 & 489 & 16.1 & $-1.82 \%$ \\
\hline T13A36 & IG110 & CRS & $\mathrm{AX}$ & Unstressed & 600 & 487 & 16.1 & $-1.91 \%$ \\
\hline T14A58 & IG110 & CRP & $\mathrm{AX}$ & Unstressed & 600 & 508 & 28.5 & $0.28 \%$ \\
\hline T14A59 & IG110 & CRP & $A X$ & Unstressed & 600 & 509 & 28.6 & $-0.13 \%$ \\
\hline $\mathrm{T} 13 \mathrm{~A} 24$ & IG110 & CRS & $\mathrm{AX}$ & Unstressed & 600 & 506 & 28.7 & $-1.07 \%$ \\
\hline T14A67 & IG110 & CRP & $\mathrm{AX}$ & Unstressed & 600 & 478 & 42.3 & $3.67 \%$ \\
\hline T14A68 & IG110 & CRP & $\mathrm{AX}$ & Unstressed & 600 & 479 & 42.6 & $4.17 \%$ \\
\hline T13A30 & IG110 & CRS & $\mathrm{AX}$ & Unstressed & 600 & 477 & 42.7 & $4.39 \%$ \\
\hline
\end{tabular}




\section{REFERENCES}

1. Campbell, A.A. and Y. Katoh, "Report on Effects of Irradiation on Material IG-110 -Prepared for Toyo Tanso Co., Ltd.", ORNL/TM-2017/705, (2017), 

ORNL/LTR-2018/511

\section{INTERNAL DISTRIBUTION}

1. Y. Katoh

2. A.A. Campbell

3. K.D. Linton

4. T.D. Burchell

5. M. Vance

\section{EXTERNAL DISTRIBUTION}

6. Mr. Masatoshi Yamaji, Toyo Tanso Co., Ltd., Telephone +81-875-83-6917, email: myamaji@toyotanso.co.jp

7. Mr. Eiji Kunimoto, Toyo Tanso Co., Ltd., Telephone +81-875-83-6917, email: e.kunimoto@toyotanso.co.jp

8. Ms. Alice Caponiti, DOE Office of Nuclear Energy, NE-20/GTN, Telephone: (301) 9036062, email: Alice.Caponiti@ nuclear.energy.gov 